\title{
Inhibitory effects of resveratrol on foam cell formation are mediated through monocyte chemotactic protein-1 and lipid metabolism-related proteins
}

\author{
WENPENG DONG $^{1 *}$, XIANYUE WANG ${ }^{1 *}$, SHENGHUI BI ${ }^{1 *}$, ZHIGUO PAN $^{2}$, SHENXI LIU $^{3}$,

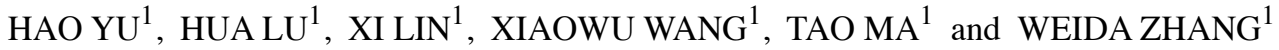 \\ ${ }^{1}$ Department of Cardiovascular Surgery, ${ }^{2}$ Intensive Care Unit and ${ }^{3}$ Information Center, \\ Guangzhou General Hospital of Guangzhou Military Command, Guangzhou, Guangdong 510010, P.R. China
}

Received September 24, 2013; Accepted February 3, 2014

DOI: $10.3892 /$ ijmm.2014.1680

\begin{abstract}
Resveratrol has been shown to exert anti-atherosclerotic effects. 5' AMP-activated protein kinase (AMPK) and monocyte chemotactic protein-1 (MCP-1) play key roles in foam cell formation, which is considered as the initiation of atherosclerosis. Thus, in this study, we investigated whether resveratrol inhibits foam cell formation by regulating lipid accumulation and inflammation. For this purpose, THP-1 cells were treated with $100 \mathrm{nM}$ phorbol 12-myristate 13-acetate (PMA) to induce their differentiation into macrophages. The macrophages were then pre-treated with $2.5 \mu \mathrm{M}$ resveratrol and subsequently with serum-free (SF) medium alone or SF medium containing lipopolysaccharide (LPS; $100 \mathrm{ng} / \mathrm{ml}$ ) and oxidized low-density lipoprotein (ox-LDL; $50 \mu \mathrm{g} / \mathrm{ml}$ ) for $24 \mathrm{~h}$ to detect foam cell formation. To detect the expression of lipid accumulation-related proteins, the macrophages were treated with resveratrol. For the detection MCP-1 expression, the macrophages were treated with LPS and resveratrol, or with resveratrol alone. We incubated the THP-1-derived macrophages in resveratrol $(2.5 \mu \mathrm{M})$ for $6 \mathrm{~h}$ in the presence or absence of $30 \mu \mathrm{M}$ compound $\mathrm{C}$ for $4 \mathrm{~h}$ to detect the influence of compound $\mathrm{C}$ on the effects of resveratrol. The foam cells were examined using Red O staining. Gene expression levels were determined by qRT-PCR, western blot analysis and ELISA; lipid analysis was carried out by high-performance liquid chromatography (HPLC). The results revealed that resveratrol effectively suppressed foam cell formation induced by LPS. Resveratrol also suppressed lipid accumulation and downregulated the mRNA expression of peroxisome prolifer-
\end{abstract}

Correspondence to: Dr Weida Zhang, Department of Cardiovascular Surgery, Guangzhou General Hospital of Guangzhou Military Command, Guangzhou, Guangdong 510010, P.R. China E-mail: weidazhanggz@163.com

*Contributed equally

Key words: atherosclerosis, THP-1 cells, resveratrol, inflammation, lipid metabolism ator-activated receptor (PPAR) $\gamma$ and PPAR $\alpha$, but had no effect on the expression of PPAR $\beta / \delta$. Resveratrol also upregulated the expression of AMPK and Silent information regulator T1 (SIRT1). However, the effects of resveratrol on SIRT1, PPAR $\gamma$ and PPAR $\alpha$ expression and lipid accumulation were reversed when the cells were pre-treated with compound C. Resveratrol downregulated the mRNA expression of MCP-1 in a dosedependent manner and LPS upregulate its expression in a time-dependent manner. MCP-1 expression induced by LPS was inhibited by resveratrol at both the transcriptional and translational level. These data suggest that resveratrol inhibits foam cell formation by regulating the expression of MCP-1 and activating the AMPK-SIRT1-PPAR signaling pathway; thus, resveratrol may be a novel therapeutic agent for atherosclerosis.

\section{Introduction}

Atherosclerosis is a chronic inflammatory disease characterized by inflammatory infiltrates and lipid accumulation (1). It is known that monocytes play an important role in the progression of the disease $(2,3)$. Monocytes first migrate into the arterial tissue in response to locally produced chemokines and then differentiate into macrophages. These macrophages act to augment the expression of several pattern recognition receptors, leading to the accumulation of cholesterol and lipids; thse cells then become foam cells (4-6). Therefore, inflammatory infiltrates and the accumulation of cholesterol and lipids in macrophages is a key point in the initiation of atherosclerosis.

Over the past decade, the search for natural compounds with the ability to prevent atherosclerosis has been a main focus for many investigators. Several lines of evidence suggest that resveratrol, a plant-derived polyphenol and phytoalexin, exhibits cardioprotective and anti-inflammatory properties (7-10). In the current study, we investigated the potential atheroprotective, anti-inflammatory and lipid-lowering effects of resveratrol. The oral administration of resveratrol has been shown to affect lipid profiles and inflammation markers (11). However, the precise mechanisms involved remain unclear.

The balanced flow of lipids into and out of macrophages is necessary to avoid lipid overload, and ultimately, foam cell formation (12). 5' AMP-activated protein kinase (AMPK) is an 
important serine/threonine kinase well known for regulating cellular energy levels by balancing nutrient availability and energy demand through its control of several proteins involved in glucose and lipid metabolism $(13,14)$. Recent evidence has shown a promising role for AMPK in the attenuation of atherosclerosis and vascular dysfunction (14). Resveratrol can activate the AMPK pathway in adipose tissue (15). In addition, it has been demonstrated that resveratrol exerts cardiometabolic effects by increasing AMPK expression and the level of Silent information regulator T1 (SIRT1) (16).

Conventional risk factors for atherosclerosis trigger an inflammatory response in the artery wall, mediated by complex molecular interactions in which chemokines play a critical role (17). Monocyte chemotactic protein-1 (MCP-1) is a potent chemoattractant for monocytes and plays a pivotal role in early atherogenesis by promoting monocyte infiltration to lesion-prone areas and penetrates between endothelial cells into the inner arterial space $(18,19)$. MCP-1 is synthesized by endothelial cells and monocytes in response to diverse stimuli, including interleukins and oxidized low-density lipoprotein (ox-LDL) (18). Lipopolysaccharide (LPS) is thought to be involved in cardiovascular disease, as it contributes to the development of arterial plaques through activated pro-inflammatory pathways by secreting cytokines, including MCP-1 $(20,21)$. The mechanisms which have been suggested to be responsible for the anti-inflammatory effects of resveratrol, include the inhibition of MCP-1 production (22).

In this study, we investigated the effects of resveratrol on foam cell formation, as well as on the expression of MCP-1 and AMPK in macrophages in order to elucidate the mechanisms involved in its anti-atherosclerotic effects. In addition, the expression of SIRT1 and nuclear peroxisome proliferatoractivated receptors (PPARs) was detected. The results of this study provide important information regarding the initiation and prevention of atherosclerosis.

\section{Materials and methods}

Materials. Resveratrol, Oil Red O and compound C were purchased from Sigma-Aldrich, St. Louis, MO, USA; antibodies directed against phospho-AMPKa (Thr172), AMPK, SIRT1 and $\beta$-actin were from Cell Signaling Technology, Danvers, MA, USA; Texas-Red-conjugated goat anti-mouse secondary antibody was obtained from Molecular Probes, Eugene, OR, USA; LPS, phorbol 12-myristate 13-acetate (PMA) and TRIzol reagent were from Sigma-Aldrich; the cDNA Synthesis kit was obtained from Fermentas, St. Leon-Rot, Germany; the SYBR Premix Ex Taq ${ }^{\mathrm{TM}}$ II kit was purchased from Takara Bio Inc., Otsu, Japan; the Human MCP-1 ELISA kit was from R\&D Systems, Minneapolis, MN, USA; the BCA kit was from Pierce, Rockford, IL, USA; and Agilent 1100 series HPLC system was from Agilent Technologies, Palo Alto, CA, USA.

Preparation of ox-LDL. Human LDL was purified from the fresh plasma of healthy donors by sequential centrifugation, according to the method described in the study by Feng et al (23) with some modifications. In order to produce ox-LDL, $200 \mu \mathrm{g} / \mathrm{ml} \mathrm{LDL}$ were exposed to $20 \mu \mathrm{M} \mathrm{CuSO}_{4}$ in phosphate-buffered saline (PBS) for oxidation and the reaction was terminated with $40 \mu \mathrm{M}$ butylhydroxytoluene in ethanol.
ox-LDL was then dialyzed against culture medium and sterilefiltered.

Cell culture. The human monocytic cell line, THP-1 (ATCC, Rockville, MD, USA), was grown in RPMI-1640 supplemented with $10 \%$ (v/v) fetal bovine serum (FBS), $0.05 \mathrm{mM} 2$-mercaptoethanol, $10 \mathrm{mM}$ HEPES, $1 \mathrm{mM}$ sodium pyruvate, $4.5 \mathrm{~g} / \mathrm{l}$ glucose and $1.5 \mathrm{~g} / \mathrm{l}$ bicarbonate at $37^{\circ} \mathrm{C}$ in an atmosphere containing $5 \% \mathrm{CO}_{2}$. The THP-1 cells were stimulated by a 48-h exposure to $100 \mathrm{nM}$ PMA to induce their differentiation into adherent macrophages. Differentiated THP-1 macrophages were extensively washed in PBS before being used in experiments.

Resveratrol treatment. A stock concentration of $100 \mathrm{mM}$ resveratrol in 50\% DMSO was produced fresh each time and diluted in culture medium to the desired concentration. The controls received the same amount of DMSO.

Foam cell formation assay. The experiments were performed in serum-free (SF) experimental medium. The THP-1 cells were pre-treated with $2.5 \mu \mathrm{M}$ of resveratrol or the control (DMSO) for $1 \mathrm{~h}$. Subsequently, both the control and resveratrol-treated groups were treated with SF medium alone or SF medium containing LPS $(100 \mathrm{ng} / \mathrm{ml})$ and ox-LDL $(50 \mu \mathrm{g} /$ $\mathrm{ml}$ ) for $24 \mathrm{~h}$. The formation of foam cells was determined by Oil Red $\mathrm{O}$ staining. The cells were fixed with $4 \%$ formaldehyde for $15 \mathrm{~min}$. Cell lipids were stained with Oil Red O ( $3 \mathrm{mg} / \mathrm{ml}$ in $60 \%$ isopropanol) for $10 \mathrm{~min}$, then observed under a microscope.

Quantitative reverse transcription PCR ( $q R T-P C R)$. Total cellular RNA was extracted from the THP-1-derived macrophages using TRIzol reagent in accordance with the manufacturer's instructions, and dissolved in nuclease-free water, prior to being reverse-transcribed to synthesize firststrand cDNA with oligo(dT) primer using the cDNA Synthesis kit. To correct for differences in cDNA loading among the samples, the target PCRs were normalized to a reference PCR involving the endogenous housekeeping gene, $\beta$-actin. Non-template controls were included for each primer pair to check for any significant levels of contaminants. A meltingcurve analysis was performed to assess the specificity of the amplified PCR products. qRT-PCR was performed using the FastStart SYBR-Green reagent kit according to the manufacturer's instructions. The reaction conditions followed the instructions provided by the manufacturer of the SYBR Premix Ex Taq II kit using gene-specific primers for MCP-1, SIRT1, PPARs and $\beta$-actin (Table I).

Lipid analysis by high-performance liquid chromatography (HPLC). Cellular total cholesterol and triglyceride contents were analyzed by lipid analysis by HPLC. Briefly, the cells were washed 3 times in PBS and lysed by the addition of $0.9 \%$ $\mathrm{NaOH}$ solution. Protein concentration was measured using the BCA kit. Masterol was used as a standard curve first, and the extraction procedure was then repeated. Samples were dissolved in $100 \mu \mathrm{l}$ of isopropanol-acetonitrile (v/v, 20:80), followed by incubation in an ultrasonic water at room temperature for $5 \mathrm{~min}$. Finally, the samples were placed in the Agilent 1100 series HPLC system. 
Table I. Primer sequences used in qRT-PCR.

\begin{tabular}{ll}
\hline Gene & \multicolumn{1}{c}{ Primer sequence $\left(5^{\prime} \rightarrow 3^{\prime}\right)$} \\
\hline$\beta$-actin & F: GATCATTGCTCCTCCTGAGC \\
& R: ACTCCTGCTTGCTGATCCAC \\
SIRT1 & F: GAGTGGCAAAGGAGCAGA \\
& R: TCTGGCATGTCCCACTATC \\
PPAR $\gamma$ & F: GCAGTGGGGATGTCTCATAATGC \\
& R: CAGGGGGGTGATGTGTTTGAA \\
PPAR $\beta / \delta$ & F: AATGCCTACCTGAAAAACTTCAAC \\
& R: GTGCACGCTGATTCCTTGT \\
PPAR $\alpha$ & F: GAGAAAGCAAAACTGAAAGCAGAGA \\
& R: GAAGGGCGGGTTATTGCTG \\
MCP-1 & F: AGCCACCTTCATTCCCCAAG \\
& R: CTCCTTGGCCACAATGGTCT \\
\hline
\end{tabular}

SIRT1, Silent information regulator T1; PPAR, peroxisome proliferator-activated receptor; MCP-1, monocyte chemotactic protein-1; F, forward, $\mathrm{R}$, reverse.

Western blot analysis. Protein concentrations were determined using bovine serum albumin (BSA) as a standard protein with the BCA protein assay. The same amounts of total proteins (15-20 $\mu \mathrm{g} /$ lane) were loaded onto each lane and transferred to nitrocellulose membranes at $80 \mathrm{~V}$ for $1 \mathrm{~h}$. After blocking for $4 \mathrm{~h}$ in $5 \%$ skim milk, the membranes were incubated overnight at $4^{\circ} \mathrm{C}$ with a $1: 1,000$ dilution of primary antibody. Following incubation with the corresponding secondary antibody, signals were detected using a chemiluminescent detection system and quantified using Quantity One analysis software.

ELISA. To evaluate the produced levels of MCP-1, the THP-1derived macrophages were pre-treated with resveratrol $(10 \mu \mathrm{M})$ for $1 \mathrm{~h}$ and then treated with LPS $(10 \mu \mathrm{M})$ for $6 \mathrm{~h}$. Supernatants from the treated cells were collected and analyzed for MCP-1 using a sandwich ELISA kit according to the manufacturer's instructions.

Data analysis. All results are expressed as the means \pm standard deviation (SD). All data were evaluated using SPSS 11.0 software. A typical image from at least 3 similar experiments is presented. Statistical analysis were carried out using t-tests. A value of $\mathrm{P}<0.05$ was considered to indicate a statistically significant difference.

\section{Results}

Resveratrol inhibits foam cell formation. We investigated foam cell formation in response to exposure to LPS. As evidenced by Oil Red $\mathrm{O}$ staining, in the presence of ox-LDL, the typical formation of foam cells was observed (Fig. 1A-II vs. A-I). Resveratrol effectively suppressed the foam cell formation induced by LPS (Fig. 1A-III vs. A-II). We investigated intracellular lipid accumulation by HPLC. LPS at $100 \mathrm{ng} / \mathrm{ml}$ without LDL loading increased lipid accumulation in the macrophages (Fig. 1B, LPS vs. SF medium, solid bars). In the presence of ox-LDL (50 $\mu \mathrm{g} / \mathrm{ml})$ loading, LPS further increased lipid accumulation in the macrophages (Fig. 1B, LPS ox-LDL vs. ox-LDL, solid bars). These observations led us to hypothesize that resveratrol may inhibit foam cell formation by regulating lipid accumulation.

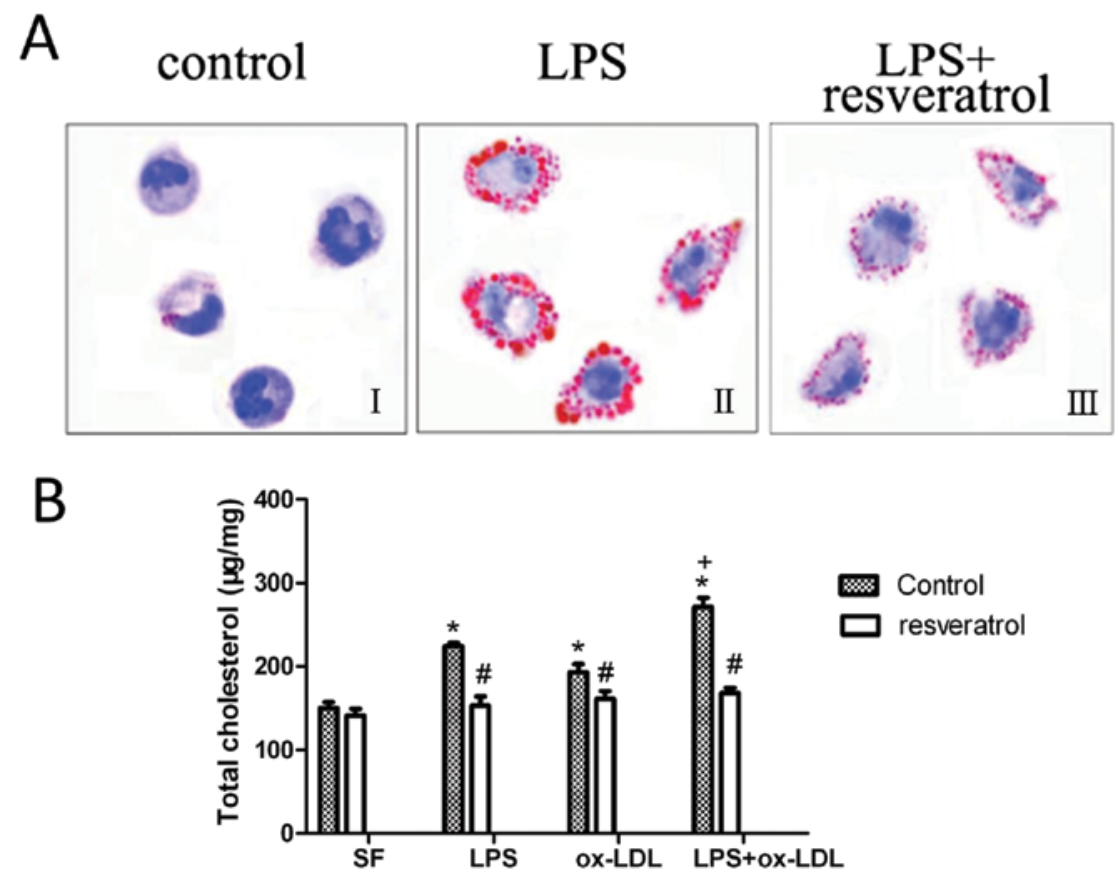

Figure 1. Resveratrol inhibits LPS-induced foam cell formation. THP-1-derived macrophages were cultured in the absence or presence of resveratrol then treated with LPS $(100 \mathrm{ng} / \mathrm{ml})$ containing ox-LDL $(50 \mu \mathrm{g} / \mathrm{ml})$ for $24 \mathrm{~h}$. (A) Foam cell was analyzed through Oil-red O staining, and photographed at a magnification of x400. (B) Cholesterol ester was assayed as described in Materials and methods. Results are described as the means \pm SD of duplicate wells of 6 experiments. ${ }^{*} \mathrm{P}<0.05$ vs. group treated with serum-free $(\mathrm{SF})$ medium; ${ }^{*} \mathrm{P}<0.05$ resveratrol vs. the corresponding control group; ${ }^{+} \mathrm{P}<0.05 \mathrm{vs}$. the ox-LDL group. 
A

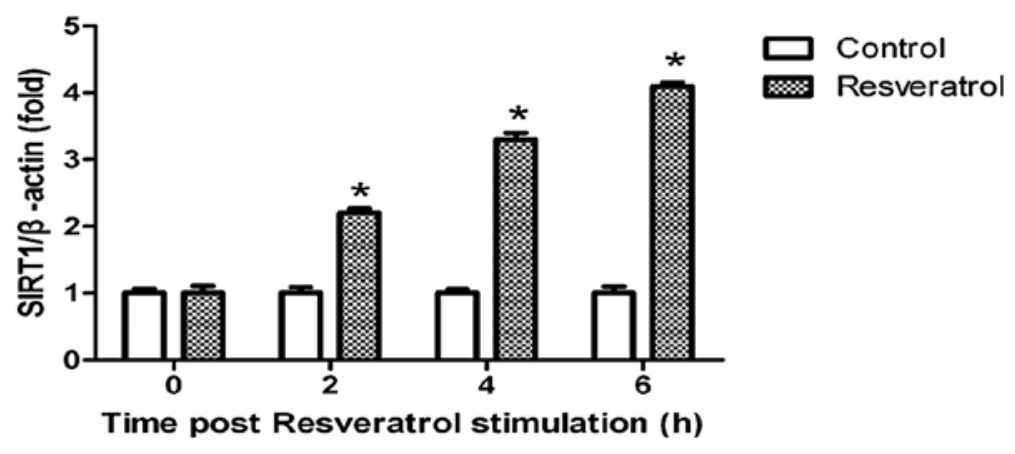

B

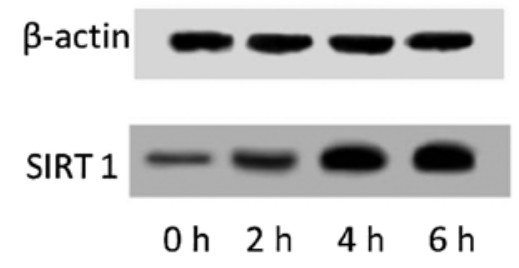

$43 \mathrm{kDa}$

$120 \mathrm{kDa}$

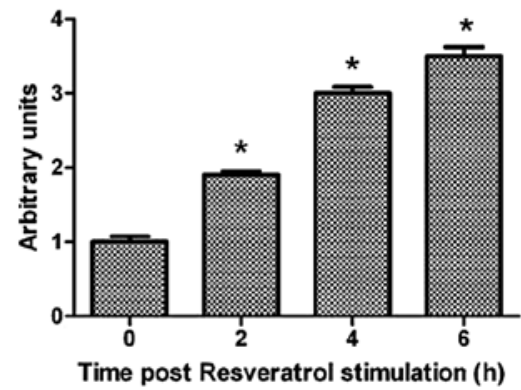

Figure 2. Effects of resveratrol on SIRT1 expression. THP-1-derived macrophages were treated (6 h) with resveratrol (2.5 $\mu \mathrm{M})$. mRNA and protein levels were examined at 0,2,4 and $6 \mathrm{~h}$ of resveratrol treatment. (A) Effect of resveratrol on SIRT1 mRNA expression. Data are the means \pm SEM; $\mathrm{n}=6$. ${ }^{*} \mathrm{P}<0.05 \mathrm{vs}$. control. (B) Effect of resveratrol on SIRT1 protein expression. ${ }^{\mathrm{P}}<0.05$ vs. control.

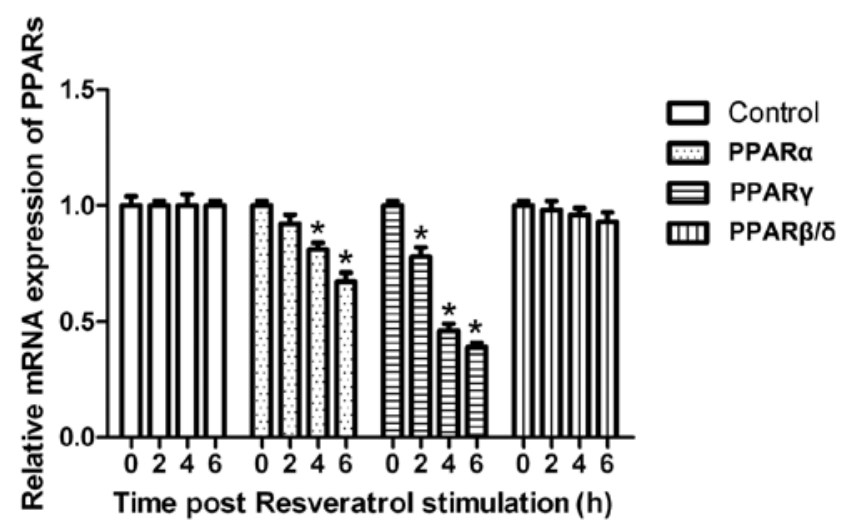

Figure 3. Effects of resveratrol on PPAR expression. Effect of resveratrol on PPAR mRNA expression, THP-1-derived macrophages were treated $(6 \mathrm{~h})$ with resveratrol $(2.5 \mu \mathrm{M})$. Data are the means $\pm \mathrm{SEM} ; \mathrm{n}=6$. ${ }^{*} \mathrm{P}<0.05$ vs. control.

Resveratrol inhibits lipid accumulation through SIRT1 and PPARs. SIRT1 reduces the accumulation of fatty acids by suppressing the expression of PPAR- $\gamma$ (24). PPAR- $\gamma$ is one of the PPAR family members, which comprises 3 isotypes: PPAR $\alpha$, PPAR $\gamma$ and PPAR $\beta / \delta$ (25), PPARs play a central role in the regulation of adipogenesis (26). Resveratrol is an activator of SIRT1 (27). Thus, this prompted us to investigate whether resveratrol regulates lipid accumulation through SIRT1-PPARs.

To investigate the effects of resveratrol on SIRT1 and PPAR expression, the THP-1-derived macrophages were treated with resveratrol $(2.5 \mu \mathrm{M})$ for $6 \mathrm{~h}$. As shown in Fig. 2A, resveratrol significantly upregulated the mRNA expression of SIRT1 $(\mathrm{P}<0.05)$, and there were analogous results obtained by western blot analysis (Fig. 2B).
We then detected the expression of PPARs. As shown in Fig. 3, resveratrol significantly downregulated the mRNA expression of PPAR $\gamma(\mathrm{P}<0.05)$. As regards PPAR $\alpha$, resveratrol also significantly downregulated the mRNA expression $(\mathrm{P}<0.05)$. By contrast, resveratrol had no effect on the mRNA expression of PPAR $\beta / \delta(\mathrm{P}<0.05)$, suggesting that resveratrol regulates lipid accumulation through SIRT1-PPARs.

Resveratrol regulates SIRT1-PPARs through AMPK. As shown by our results, resveratrol regulates the expression of SIRT1 and PPARs; however, the mechanisms involved are not yet clear. AMPK, which acts upstream of SIRT1, controls several proteins involved in glucose and lipid metabolism $(13,14)$. The activation of AMPK activates catabolic pathways, generating ATP, and 'switches off' a number of processes that consume ATP, such as fatty acid, protein, or cholesterol synthesis $(28,29)$; AMPK also enhances SIRT1 activity $(30,31)$.

To investigate the effects of resveratrol on AMPK, the THP-1-derived macrophages were treated with resveratrol $(2.5 \mu \mathrm{M})$ for $6 \mathrm{~h}$. The phosphorylated isoform is the active AMPK form; thus, we determined the phosphorylated AMPK/ total AMPK protein ratio. Resveratrol significantly increased the phosphorylated AMPK/total AMPK protein ratio; this increase demonstrated that AMPK was significantly activated by resveratrol (Fig. 4), suggesting that resveratrol regulates SIRT1-PPARs through AMPK.

Effects of AMPK inhibition on SIRT1 and PPAR expression and lipid accumulation. To verify our hypothesis, we evaluated the effects of AMPK inhibition (using compound C) on SIRT1 and PPAR expression and lipid accumulation following incubation of THP-1-derived macrophages in resveratrol $(2.5 \mu \mathrm{M})$ for $6 \mathrm{~h}$ in the presence or absence of $30 \mu \mathrm{M}$ compound $\mathrm{C}$ 


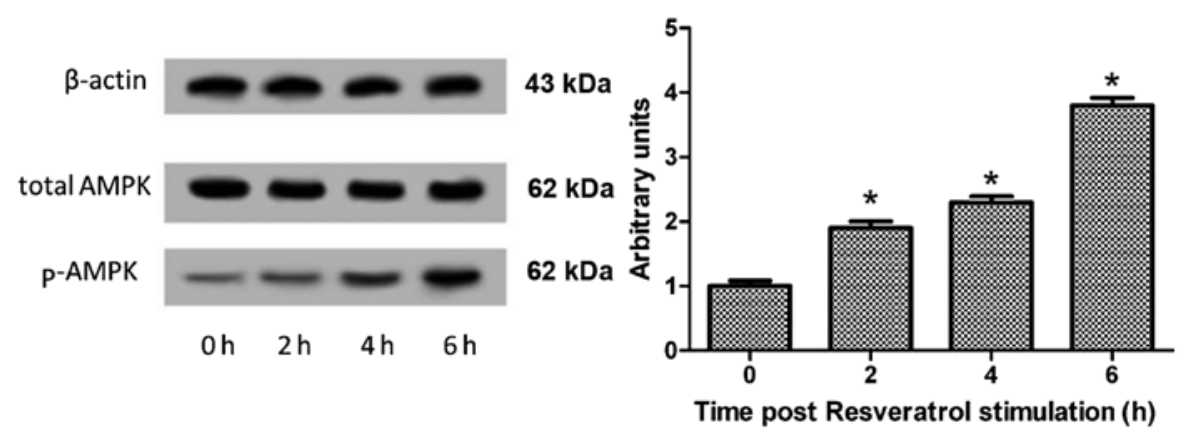

Figure 4. Effects of resveratrol on activation of AMPK. Effect of resveratrol on activation of AMPK. THP-1-derived macrophages were treated (6 h) with resveratrol $(2.5 \mu \mathrm{M})$. Phosphorylated (p)-AMPK and total AMPK expression was examined at $0,2,4$ and $6 \mathrm{~h}$ of resveratrol treatment. ${ }^{*} \mathrm{P}<0.05 \mathrm{vs}$. control.
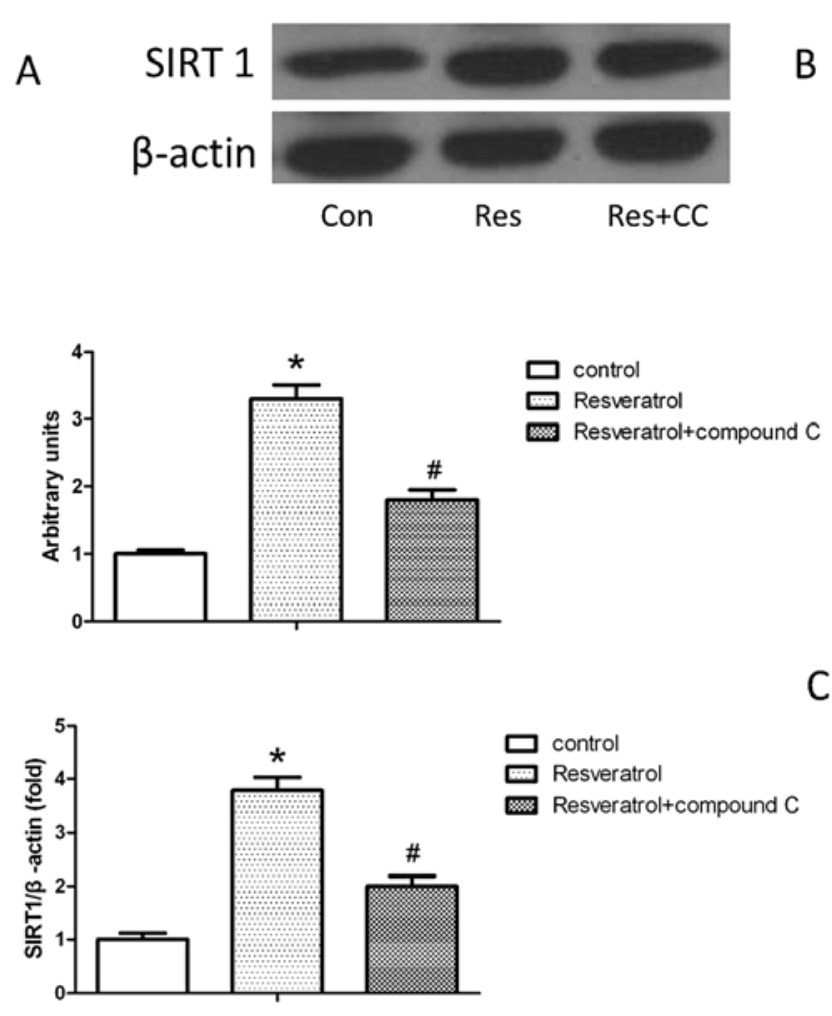

B

C
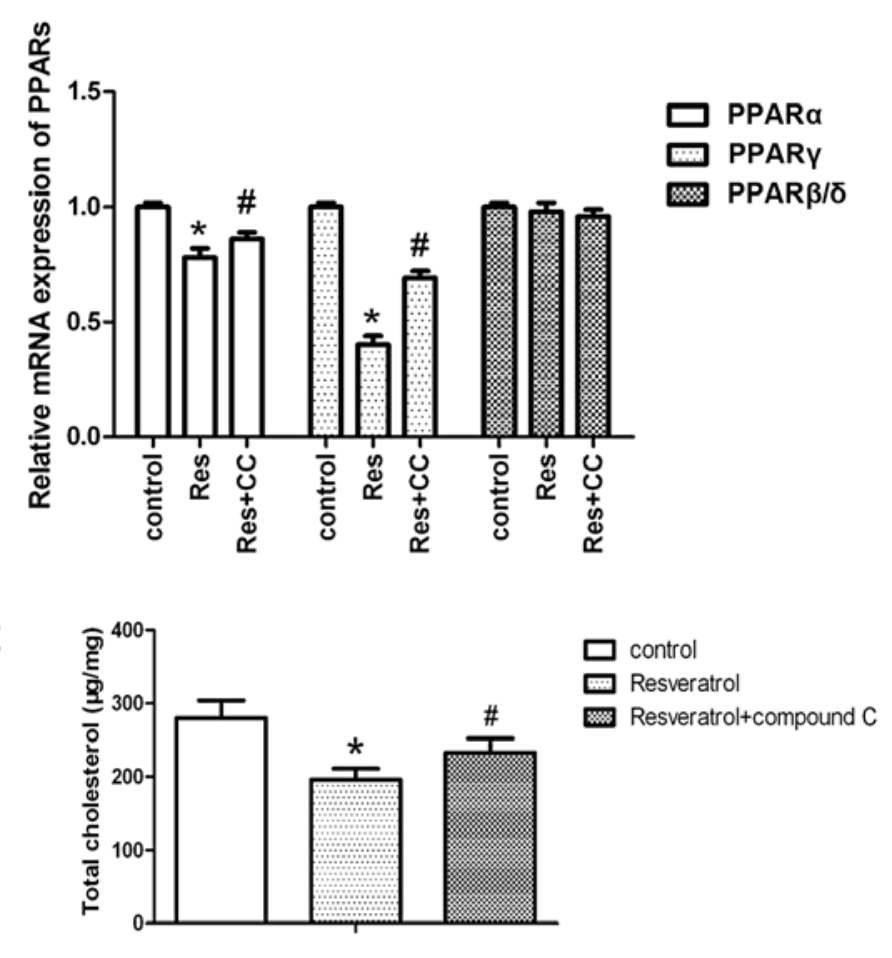

Figure 5. Effect of the AMPK inhibition in the SIRT1, PPAR expression and lipid accumulation. THP-1-derived macrophages incubated in resveratrol (2.5 $\mu$ M) for $6 \mathrm{~h}$ in the presence or absence of $30 \mu \mathrm{M}$ compound $\mathrm{C}$ for $4 \mathrm{~h}$. (A) Effect of compound $\mathrm{C}$ on the expression of SIRT1. (B) Effect of compound C in the expression of PPARs. (C) Effect of compound $\mathrm{C}$ on lipid accumulation. ${ }^{\mathrm{P}} \mathrm{P}<0.05$ vs. control; ${ }^{*} \mathrm{P}<0.05$ vs. resveratrol.

for $4 \mathrm{~h}$. Our results revealed that pre-treatment with $30 \mu \mathrm{M}$ compound $\mathrm{C}$ for $4 \mathrm{~h}$ inhibited the resveratrol-induced increase in SIRT1 expression and blocked the suppression of PPAR $\gamma /$ PPAR $\alpha$ expression by resveratrol (Fig. 5A and B). We also examined lipid accumulation; the suppression of lipid accumulation following treatment with resveratrol was reversed when the cells were pre-treated with $30 \mu \mathrm{M}$ compound C (Fig. 5C).

Resveratrol blocks LPS-induced MCP-1 expression. Given the well-documented anti-inflammatory effects of resveratrol, we focused on the effects of resveratrol on LPS-induced MCP-1 expression in THP-1-derived macrophages. Resveratrol downregulated the mRNA expression of MCP-1 in a dosedependent manner (Fig. 6A). As shown in Fig. 6B, MCP-1 mRNA expression increased following treatment with LPS in a time-dependent manner. Pre-treatment with $10 \mu \mathrm{M}$ resve- ratrol markedly inhibited the mRNA expression of MCP-1 induced by LPS (Fig. 6C). The induced expression of MCP-1 was determined by ELISA (Fig. 7). Compared to the control group, LPS induced a statistically significant upregulation of MCP-1 expression $(\mathrm{P}<0.05)$, and resveratrol downregulated the LPS-induced MCP-1 expression. MCP-1 expression which was induced by LPS was inhibited by resveratrol at both the transcriptional and translational level. These results demonstrate that resveratrol inhibits foam cell formation by regulating inflammatory cytokine (MCP-1) production.

\section{Discussion}

Cardiovascular disease, which is currently the leading cause of death and illness in developed countries, is a preeminent health issue worldwide (32). Atherosclerosis, a progressive 

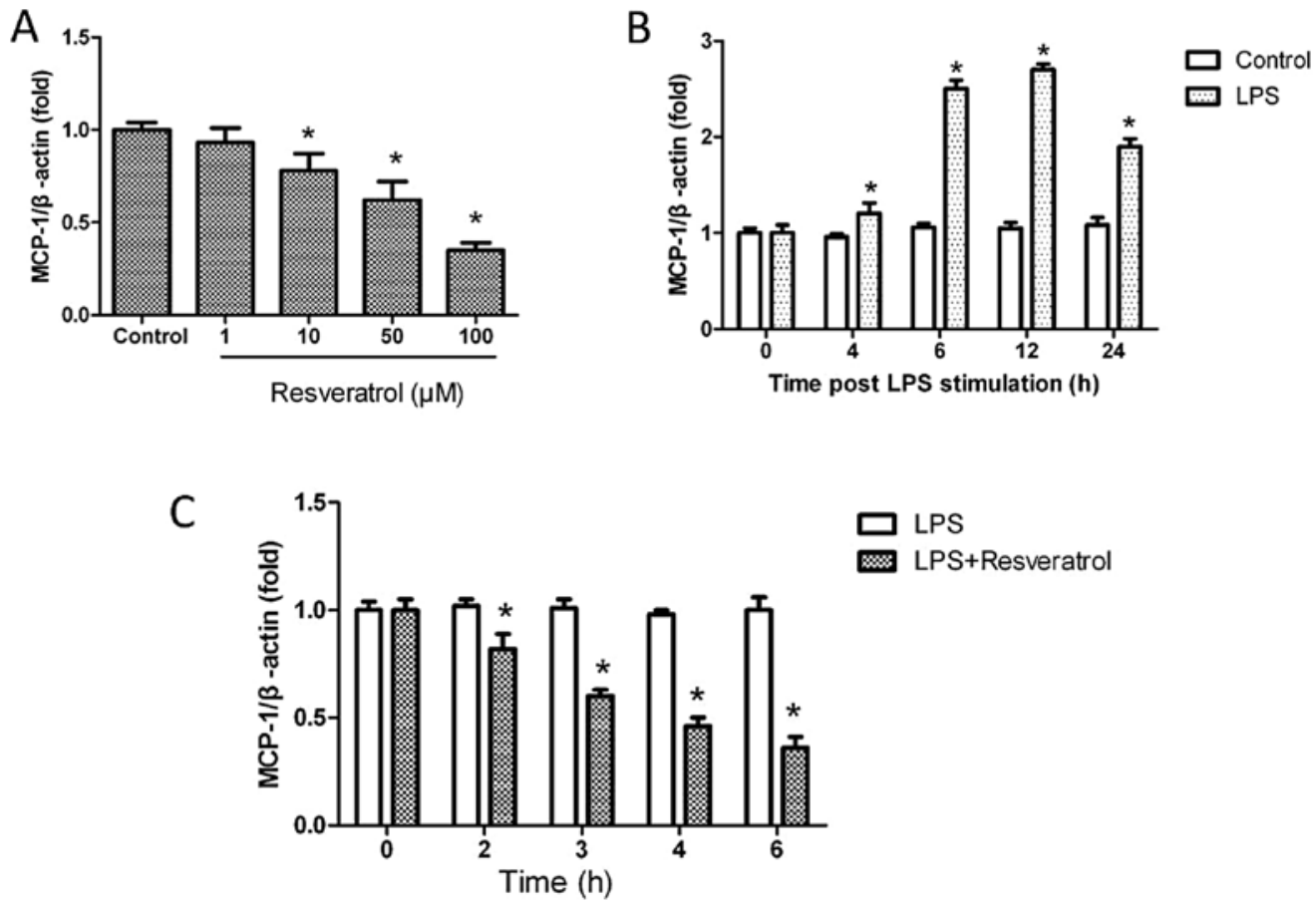

treated $(6 \mathrm{~h})$ with resveratrol $(1-100 \mu \mathrm{M})$. (B) LPS-induced MCP-1 expression, THP-1-derived macrophages were treated (24 h) with LPS (10 $\mu \mathrm{g} / \mathrm{ml})$. (C) Effect of resveratrol on LPS-induced MCP-1 expression, THP-1-derived macrophages were pre-treated with resveratrol (10 $\mu \mathrm{M})$ for $1 \mathrm{~h}$ then treated with LPS (10 $\mu \mathrm{M})$ for $6 \mathrm{~h}$. Data are the means $\pm \mathrm{SEM} ; \mathrm{n}=6 .{ }^{*} \mathrm{P}<0.05$ vs. control.

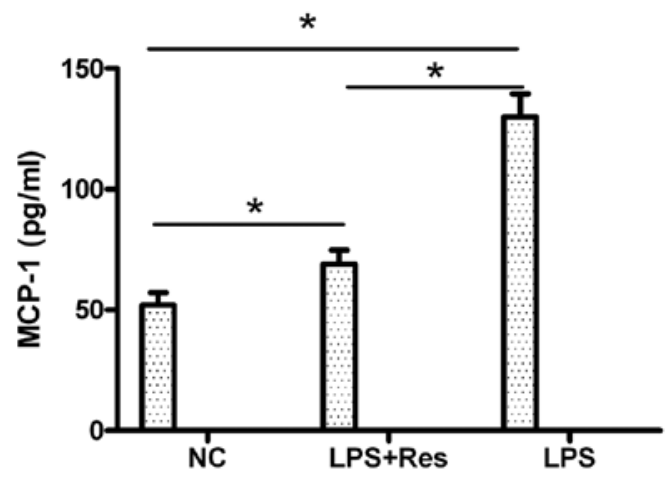

Figure 7. Effect of resveratrol on LPS-induced secretion of MCP-1. THP-1derived macrophages were pretreated with resveratrol $(10 \mu \mathrm{M})$ for $1 \mathrm{~h}$ then treated with LPS $(10 \mu \mathrm{M})$ for $6 \mathrm{~h}$. The concentration of MCP-1 in the culture medium was determined by ELISA. Results are the means $\pm \mathrm{SD}$. ${ }^{*} \mathrm{P}<0.05$ compared to control cells (NC).

inflammatory disease, produces arterial plaques characterized by inflammatory infiltrates, lipid accumulation, cell death and fibrosis $(2,3,33)$. Monocytes play an important role in the progression of the disease. Monocytes first migrate, and then differentiate into macrophages. Inflammatory infiltrates and the accumulation of cholesterol and lipids in macrophages allows them to become foam cells; this is a key point in the initiation of atherosclerosis.

Resveratrol is a polyphenol found in grapes, berries and peanuts. It inhibits macrophage activation (34), one of the most important steps in atherosclerosis, as well as lipid accumulation $(34,35)$. In addition, resveratrol has been suggested to exert anti-atherosclerotic effects (36). However, the precise mechanisms responsible for the anti-atherosclerotic effects of resveratrol remain unclear.

In this study, we found that THP-1-derived macrophages treated with LPS and ox-LDL together resulted in the typical formation of foam cells and resveratrol effectively suppressed the foam cell formation induced by LPS. Based on our findings, we suggest that resveratrol exerts anti-atherosclerotic effects by suppressing foam cell formation. Experiments on the effects of lipid deposition in THP-1-derived macrophages treated with resveratrol, revealed that the cellular total cholesterol content was suppressed by resveratrol, suggesting that resveratrol protects against atherosclerosis by inhibiting lipid accumulation.

AMPK regulates cellular energy levels by balancing nutrient availability and energy demand through its control of several proteins involved in glucose and lipid metabolism (13). SIRT1 is one of the 7 mammalian homologs of the Sir2 family that catalyzes NAD ${ }^{+}$-dependent protein deacetylation (37). Both AMPK and SIRT1 have emerged as interesting targets as they are heavily involved in catabolic metabolism, mitochondrial activation, angiogenesis and enhanced cell survival (37-40). PPARs are central regulators of adipogenesis (25). Previous studies have shown that resveratrol negatively modulates PPAR $\gamma$ protein levels in 3T3-L1 adipocytes (41) and it is known that resveratrol affects both SIRT1 and AMPK (42). The data from these studies, as well as ours, suggest that resveratrol regulates lipid metabolism through the AMPK-SIRT1-PPAR signaling pathway.

In this study, we found that AMPK was significantly activated by resveratrol and that resveratrol markedly upregulated the expression of SIRT1 both at the mRNA and protein level. In addition, resveratrol markedly downregulated the mRNA 
expression of PPAR $\gamma$ and PPAR $\alpha$, but not that of PPAR $\beta / \delta$. When the cells were pre-treated with the AMPK inhibitor, compound C, the effects of resveratrol on SIRT1, PPAR $\gamma$ and PPAR $\alpha$ expression, as well as on lipid accumulation were reversed, suggesting that resveratrol suppresses lipid accumulation through the AMPK-SIRT1-PPAR $\gamma / \mathrm{PPAR} \alpha$ signaling pathway.

Inflammatory infiltrates in macrophages are a key to foam cell formation. Thus, we hypothesized that resveratrol may suppress foam cell formation by regulating inflammatory cytokines. We selected MCP-1, a potent chemoattractant for monocytes. MCP-1 has been shown to be overexpressed in human and experimental atheroma, and can recruit mononuclear phagocytes that characteristically accumulate in the nascent atheroma $(32,43)$. A recent study demonstrated that LPS induces the expression of MCP-1 (44). Despite the evidence of a prominent role of MCP-1 in the development of atherosclerosis, and although resveratrol has been shown to inhibit the production of various types of inflammatory cytokines $(34,45)$, few studies have investigated the direct effects of resveratrol on MCP-1. In this study, we found that resveratrol downregulated the expression of MCP-1 in a dose-dependent manner in THP-1-derived macrophages and that LPS induced MCP-1 expression in THP-1-derived macrophages in a time-dependent manner. Most importantly, we found that pre-treatment of THP-1-derived macrophages with resveratrol significantly blocked MCP-1 mRNA expression induced by LPS. Therefore, we speculate that resveratrol plays a role in anti-atherosclerosis by inhibiting the expression of MCP-1.

In conclusion, our results demonstrate that resveratrol suppresses the foam cell formation induced by LPS. Resveratrol inhibits foam cell formation by regulating the expression of the inflammatory cytokine, MCP-1, and by activating the AMPK-SIRT1-PPAR signaling pathway. These results suggest that resveratrol may be a novel therapeutic agent for atherosclerosis.

\section{Acknowledgements}

This study was supported by a grant from the Natural Science Foundation of China (no. 81200633).

\section{References}

1. Andersson J, Libby P and Hansson GK: Adaptive immunity and atherosclerosis. Clin Immunol 134: 33-46, 2010.

2. Hansson GK: Inflammation, atherosclerosis, and coronary artery disease. N Engl J Med 352: 1685-1695, 2005.

3. Weber C, Zernecke A and Libby P: The multifaceted contributions of leukocyte subsets to atherosclerosis: lessons from mouse models. Nat Rev Immunol 8: 802-815, 2008.

4. Boring L, Gosling J, Cleary M and Charo IF: Decreased lesion formation in CCR $2^{-1-}$ mice reveals a role for chemokines in the initiation of atherosclerosis. Nature 394: 894-897, 1998.

5. Gu L, Okada Y, Clinton SK, et al: Absence of monocyte chemoattractant protein-1 reduces atherosclerosis in low density lipoprotein receptor-deficient mice. Mol Cell 2: 275-281, 1998.

6. Edfeldt K, Swedenborg J, Hansson GK and Yan ZQ: Expression of toll-like receptors in human atherosclerotic lesions: a possible pathway for plaque activation. Circulation 105: 1158-1161, 2002.

7. Fan EG, Zhang LJ, Jiang S and Bai YH: Beneficial effects of resveratrol on atherosclerosis. J Med Food 11: 610-614, 2008.

8. Palmieri D, Pane B, Barisione C, et al: Resveratrol counteracts systemic and local inflammation involved in early abdominal aortic aneurysm development. J Surg Res 171: e237-246, 2011.
9. Prasad K: Natural products in regression and slowing of progression of atherosclerosis. Curr Pharm Biotechnol 11: 794-800, 2010.

10. Zhu X, Liu Q, Wang M, et al: Activation of Sirt1 by resveratrol inhibits TNF-alpha induced inflammation in fibroblasts. PLoS ONE 6: e27081, 2011.

11. Militaru C, Donoiu I, Craciun A, Scorei ID, Bulearca AM and Scorei RI: Oral resveratrol and calcium fructoborate supplementation in subjects with stable angina pectoris: effects on lipid profiles, inflammation markers, and quality of life. Nutrition 29: 178-183, 2013.

12. Voloshyna I, Hai O, Littlefield MJ, Carsons S and Reiss AB: Resveratrol mediates anti-atherogenic effects on cholesterol flux in human macrophages and endothelium via PPAR gamma and adenosine. Eur J Pharmacol 698: 299-309, 2013.

13. Vingtdeux V, Chandakkar P, Zhao H, Davies P and Marambaud P: Small-molecule activators of AMP-activated protein kinase (AMPK), RSVA314 and RSVA405, inhibit adipogenesis. Mol Med 17: 1022-1030, 2011.

14. Fullerton MD, Steinberg GR and Schertzer JD: Immunometabolism of AMPK in insulin resistance and atherosclerosis. Mol Cell Endocrinol 366: 224-234, 2013.

15. Siriwardhana N, Kalupahana NS, Cekanova M, LeMieux M, Greer B and Moustaid-Moussa N: Modulation of adipose tissue inflammation by bioactive food compounds. J Nutr Biochem 24: 613-623, 2013.

16. Crandall JP and Barzilai N: Exploring the promise of resveratrol: where do we go from here? Diabetes 62: 1022-1023, 2013.

17. Coll B, Alonso-Villaverde C and Joven J: Monocyte chemoattractant protein-1 and atherosclerosis: is there room for an additional biomarker? Clin Chim Acta 383: 21-29, 2007.

18. Charo IF and Taubman MB: Chemokines in the pathogenesis of vascular disease. Circ Res 95: 858-866, 2004.

19. McLaren JE, Michael DR, Ashlin TG and Ramji DP: Cytokines, macrophage lipid metabolism and foam cells: implications for cardiovascular disease therapy. Prog Lipid Res 50: 331-347, 2011.

20. Kallio KA, Buhlin K, Jauhiainen M, et al: Lipopolysaccharide associates with pro-atherogenic lipoproteins in periodontitis patients. Innate Immun 14: 247-253, 2008.

21. Vink A, Schoneveld AH, van der Meer JJ, et al: In vivo evidence for a role of toll-like receptor 4 in the development of intimal lesions. Circulation 106: 1985-1990, 2002.

22. Park D-W, Kim J-S, Chin B-R and Baek S-H: Resveratrol inhibits inflammation induced by heat-killed Listeria monocytogenes. J Med Food 15: 788-794, 2012.

23. Feng X, Zhang Y, Xu R, et al: Lipopolysaccharide up-regulates the expression of Fcalpha/mu receptor and promotes the binding of oxidized low-density lipoprotein and its IgM antibody complex to activated human macrophages. Atherosclerosis 208: 396-405, 2010.

24. Winnik S, Stein S and Matter CM: SIRT1 - an anti-inflammatory pathway at the crossroads between metabolic disease and atherosclerosis. Curr Vasc Pharmacol 10: 693-696, 2012.

25. Semple RK, Chatterjee VK and O'Rahilly S: PPAR gamma and human metabolic disease. J Clin Invest 116: 581-589, 2006.

26. Costa Cdos S, Rohden F, Hammes TO, et al: Resveratrol upregulated SIRT1, FOXO1, and adiponectin and downregulated PPARgamma1-3 mRNA expression in human visceral adipocytes. Obes Surg 21: 356-361, 2011.

27. Nimmagadda VK, Bever CT, Vattikunta NR, et al: Overexpression of SIRT1 protein in neurons protects against experimental autoimmune encephalomyelitis through activation of multiple SIRT1 targets. J Immunol 190: 4595-4607, 2013.

28. Cantó C, Jiang LQ, Deshmukh AS, et al: Interdependence of AMPK and SIRT1 for metabolic adaptation to fasting and exercise in skeletal muscle. Cell Metab 11: 213-219, 2010.

29. Hardie DG: AMP-activated/SNF1 protein kinases: conserved guardians of cellular energy. Nat Rev Mol Cell Biol 8: 774-785, 2007.

30. Canto C, Gerhart-Hines Z, Feige JN, et al: AMPK regulates energy expenditure by modulating $\mathrm{NAD}^{+}$metabolism and SIRT1 activity. Nature 458: 1056-1060, 2009.

31. Costford SR, Bajpeyi S, Pasarica M, et al: Skeletal muscle NAMPT is induced by exercise in humans. Am J Physiol Endocrinol Metab 298: E117-E126, 2010.

32. Libby P: Inflammation in atherosclerosis. Nature 420: 868-874, 2002.

33. Hansson GK and Libby P: The immune response in atherosclerosis: a double-edged sword. Nat Rev Immunol 6: 508-519, 2006. 
34. Park D-W, Baek K, Kim J-R, et al: Resveratrol inhibits foam cell formation via NADPH oxidase 1-mediated reactive oxygen species and monocyte chemotactic protein-1. Exp Mol Med 41: 171-179, 2009.

35. Li H, Song Y, Li F, et al: Identification of lipid droplet-associated proteins in the formation of macrophage-derived foam cells using microarrays. Int J Mol Med 26: 231-239, 2010.

36. Hou X, Xu S, Maitland-Toolan KA, et al: SIRT1 regulates hepatocyte lipid metabolism through activating AMP-activated protein kinase. J Biol Chem 283: 20015-20026, 2008.

37. Lagouge M, Argmann C, Gerhart-Hines Z, et al: Resveratrol improves mitochondrial function and protects against metabolic disease by activating SIRT1 and PGC-1alpha. Cell 127 1109-1122, 2006.

38. Rodgers JT, Lerin C, Haas W, Gygi SP, Spiegelman BM and Puigserver P: Nutrient control of glucose homeostasis through a complex of PGC-1alpha and SIRT1. Nature 434: 113-118, 2005.

39. Reverchon M, Cornuau M, Cloix L, et al: Visfatin is expressed in human granulosa cells: regulation by metformin through AMPK/SIRT1 pathways and its role in steroidogenesis. Mol Hum Reprod 19: 313-326, 2013.
40. Morita Y, Wada-Hiraike O, Yano T, et al: Resveratrol promotes expression of SIRT1 and StAR in rat ovarian granulosa cells: an implicative role of SIRT1 in the ovary. Reprod Biol Endocrinol 10: 14, 2012.

41. Picard F, Kurtev M, Chung N, et al: Sirt1 promotes fat mobilization in white adipocytes by repressing PPAR-gamma. Nature 429: 771-776, 2004.

42. Baur JA, Pearson KJ, Price NL, et al: Resveratrol improves health and survival of mice on a high-calorie diet. Nature 444: 337-342, 2006.

43. von Grote EC, Venkatakrishnan V, Duo J and Stenken JA: Long-term subcutaneous microdialysis sampling and qRT-PCR of MCP-1, IL-6 and IL-10 in freely-moving rats. Mol Biosyst 7: 150-161, 2011.

44. Wang J, Si Y, Wu C, et al: Lipopolysaccharide promotes lipid accumulation in human adventitial fibroblasts via TLR4-NFkappaB pathway. Lipids Health Dis 11: 139, 2012.

45. Culpitt SV, Rogers DF, Fenwick PS, et al: Inhibition by red wine extract, resveratrol, of cytokine release by alveolar macrophages in COPD. Thorax 58: 942-946, 2003. 